\title{
Power Optimization Configuration for Piezoelectric Cantilever Arrays
}

\author{
Bong Yu Jing ${ }^{1}$ and Kok Swee Leong ${ }^{1}$ \\ ${ }^{1}$ Faculty of Electronic and Computer Engineering, Universiti Teknikal Malaysia Melaka, Melaka, Malaysia
}

\begin{abstract}
This paper investigates the changes in the output of the piezoelectric cantilever arrays when connected in different configurations. In this research matching load resistance determined and optimum output was measured by connecting the piezoelectric cantilever arrays to resistance ranging from $10 \Omega$ to $1 \mathrm{M} \Omega$ while excited by constant vibration source at frequency of $300 \mathrm{~Hz}$ and acceleration of $1-\mathrm{g}$ level. The result shows that matching load resistance for one single piezoelectric cantilever is $13 \mathrm{~K} \Omega$. When two, three and four cantilevers are connected in series, the matching load resistance is $26 \mathrm{k} \Omega, 39 \mathrm{k} \Omega$ and $52 \mathrm{k} \Omega$ respectively. While in parallel connection, matching load resistance reduced to $6.5 \mathrm{k} \Omega, 4.5 \mathrm{k} \Omega$ and $3.5 \mathrm{k} \Omega$ for two, three, and four connected cantilevers respectively. In series configuration, the voltage output produced is much higher as compared to the piezoelectric cantilever arrays that are connected in parallel connection. The voltage output of the piezoelectric cantilever increased from $3.41 \mathrm{~V}$ to $6.09 \mathrm{~V}$ when it is connected in series configuration with same polarity. Whereas in term of power output, piezoelectric cantilever arrays in parallel configuration produce higher power output as compared to piezoelectric cantilever arrays in series connection. The maximum power increased from $272 \mu \mathrm{W}$ to $521 \mu \mathrm{W}$ when two cantilevers are connected in parallel configuration with same polarity.
\end{abstract}

\section{Introduction}

Current researches and applications areas for piezoelectric materials are vast. The research stretches from the properties of piezoelectric materials, manufacturing, up to measurement techniques and practical applications; while in application wise, it is extended from sensor, actuator, transducer and now micro-power generator [1-3]. Recent researches had shown increasing interest in looking into methods to improve the performance of piezoelectric micro-power generator. Examples of the recent researches are the vibration-based MEMS piezoelectric energy harvester and power conditioning circuit which uses a MEMS piezoelectric power generator array for vibration energy harvesting [4]; wide-band energy harvesting using piezoelectric multi-cantilever which demonstrate the potential of improving the output of the piezoelectric when connected in alternating polarities [5]; and piezoelectric, solar and thermal energy harvesting for hybrid low-power generator systems with thin-film batteries which developed a hybrid power generator and storage system that has better functionality and robustness [6]. It is expected in the near future, piezoelectric material could be the replacement of battery to create a sustainable system.

This paper investigates the changes in the output of the piezoelectric cantilever arrays when connected in different configurations. In this research, piezoelectric cantilevers are connected in four configurations which are series with same polarity, series with alternating polarities, parallel with same polarity, and parallel with alternating polarities to observe the maximum power produced from each configuration. Matching load resistances were also identified to make sure optimum power was measured.

\section{Experimental Set-up}

A pre-mounted and wired standard quick-mount bending piezoelectric generator (Q220-A4-303YB) from Piezo Systems Inc [7], is used in this research as the test subject. The dimension of the piezoelectric cantilever is as shown in Fig. 1. The illustration of the experiment set-up is as shown in Fig. 2. Electrodynamics shaker was used to mimic the ambient vibration source in order to excite the cantilever, whereas, oscilloscope was used to measure the output of the piezoelectric cantilever.

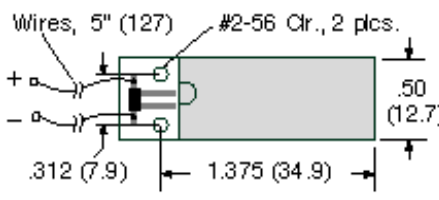

(a) Top View

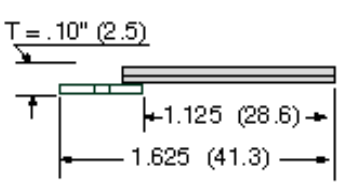

(b) Side View
Figure 1. Dimension of Piezoelectric Cantilever (Q220-A4303YB) [7].

The matching load resistance of the cantilever can be identified by connecting the piezoelectric cantilever, named CL for reference convenient, to resistance from 
the resistance decade box ranging from $10 \Omega$ to $1 \mathrm{M} \Omega$ while excited by vibration source fixed at the resonant frequency of the cantilever $(300 \mathrm{~Hz})$ and constant magnitude $1-\mathrm{g}(9.81 \mathrm{~m} / \mathrm{s} 2)$. The voltage output obtained from the piezoelectric cantilever when connected to various resistance values is observed and recorded in Fig. 4. The graph in term of voltage output is then converted to power output with the intention to identify the matching load resistance of the cantilever. The calculated result in term of power is recorded in Fig. 5.

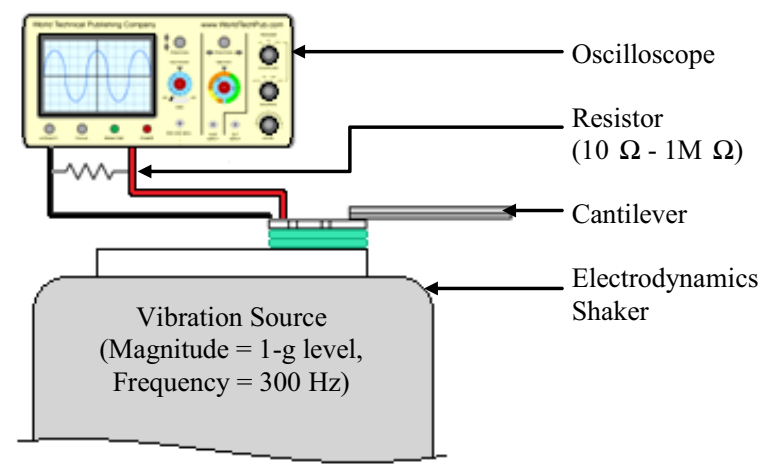

Figure 2. Illustration of the Experiment Set-up.

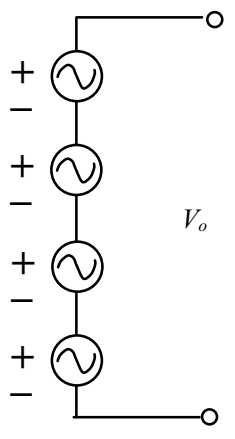

(a)

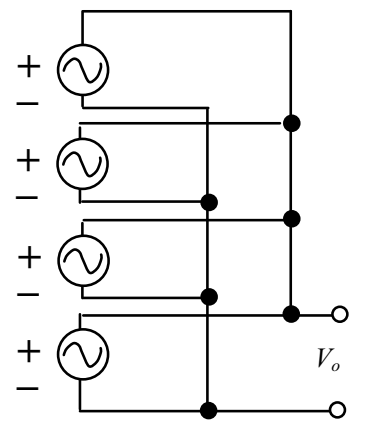

(c) (b)

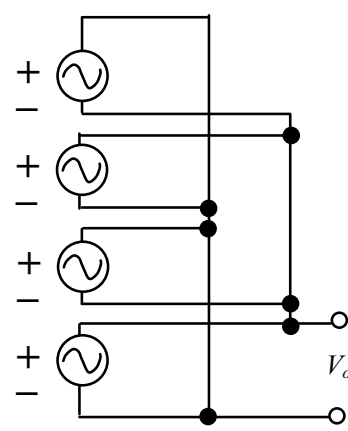

(d)

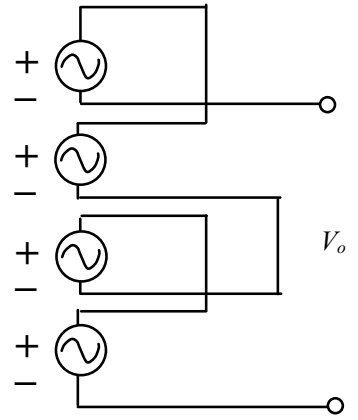

Figure 3. Illustrations for the type of Connections: (a) series, (b) series-alternating, (c) parallel and (d) parallel-alternating. [5].

In order to determine the maximum power of the piezoelectric cantilever arrays, the experiment is then continued by replacing the single piezoelectric cantilever with an array of cantilevers connected in four different configurations as shown in Fig. 3. The connections included series with same polarity, series with alternating polarities, parallel with same polarity, and parallel with alternating polarities. Piezoelectric cantilever is added one after another in order to examine the effect of the number of the connected cantilevers towards the total load resistance of the array. Three same sized cantilevers are used, named CL1, CL2 and CL3 for reference convenient. The output responses of the cantilever arrays in term of voltage and power are recorded and discussed in Section III.

\section{Experimental Result}

The output response in term of voltage and power for single piezoelectric cantilever $\mathrm{CL}$ is as shown in Fig.4 and Fig. 5 respectively. The graph in Fig. 4 shows that the output voltage produced increase in respect of the increasing of load resistance value. The output voltage increase slowly during low load resistance, then increased rapidly after $5 \mathrm{k} \Omega$, then start to saturate at high load resistance. The maximum output voltage for CL is $3.41 \mathrm{~V}$. After converting the graph into power value, peak is formed as shown in Fig. 5. The result shows that peak formed at matching load resistance of $13 \mathrm{k} \Omega$ for CL with maximum of $272.25 \mu \mathrm{W}$. This power value is the maximum power that Cantilever CL could achieve for vibration of $1-\mathrm{g}$ level at frequency of $300 \mathrm{~Hz}$.

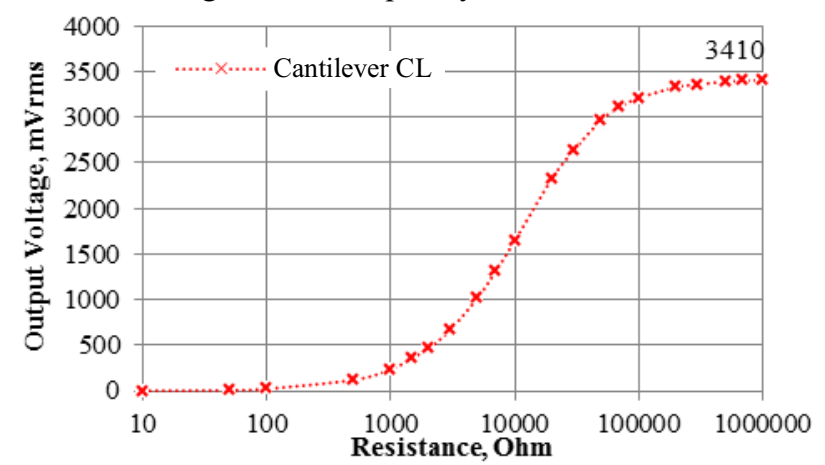

Figure 4. Output Responses (Voltage) for Cantilever CL with Varies Load Resistance.

The experiment is then continued by replacing the single piezoelectric cantilever with piezoelectric cantilever arrays connected in series with same polarity, series with alternating polarities, parallel with same polarity, and parallel with alternating polarities configurations.

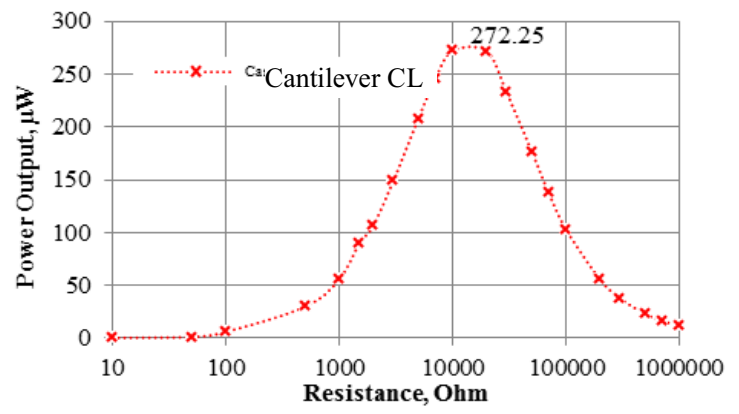

Figure 5. Output Responses (Power) for Cantilever CL with Varies Load Resistance.

\subsection{Series Configuration with Same Polarity}


The series with same polarity connected cantilevers are observed under various resistances in this subsection. The result in term of voltage output is shown in Fig. 6 while for the result in term of power value is shown in Fig. 7.

Similar to result in Fig. 4, the output increases slowly when low load resistance is connected to the cantilevers. It started to increase rapidly after $3 \mathrm{k} \Omega$ and started to saturate when connected to high load resistance. Notice that there is significant increase in maximum voltage output when cantilevers are connected as compared to the maximum voltage output produced by CL alone. Two series with same polarity connected cantilevers, CL and CL1 produced maximum voltage of $5.49 \mathrm{~V}$. While three series with same polarity connected cantilevers, CL, CL1, and CL2 produced maximum voltage of 5.73V. As four series with same polarity connected cantilevers, CL, CL1, CL2, and CL3 produced maximum voltage of $6.09 \mathrm{~V}$. The reason of the increasing of voltage output is due to the source are connected in series connection. When the sources are connected in series, the output voltage boosted up since the total voltage output would be same as adding all voltage output produced by each source.

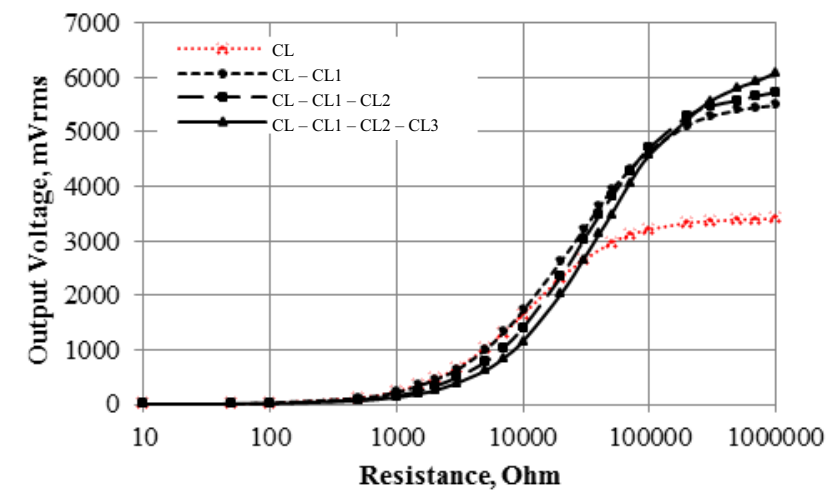

Figure 6. Output Responses (Voltage) toward Varies of Load Resistance for Cantilevers Connected in Series with Same Polarity Connection.

However, in series connection, the current output is expected to be lower and this would definitely affect the power output of the connected cantilevers. Based on the result obtained in Fig. 7, where the value is converted into power output, a peak is shown in each graph. The power generated is optimum at $346 \mu \mathrm{W}$ for two connected cantilevers during $26 \mathrm{k} \Omega$ load resistance, reduced to $302 \mu \mathrm{W}$ for three connected cantilevers during $39 \mathrm{k} \Omega$ load resistance, and further reduces to $245 \mu \mathrm{W}$ for four connected cantilevers during $52 \mathrm{k} \Omega$ load resistance.

The decreasing of maximum power is due to the fact that current value drops when connected in series because of the coupling effect from the piezoelectric cantilevers. Due to the fact that piezoelectric is conserver, the charge produced by the piezoelectric in direct piezoelectric effect will be dissipate through another conservative piezoelectric cantilever as there is no isolation between the cantilevers when connected together in series connection. Therefore adding more cantilevers in series with same polarity connection is not helpful in term of power output since it reduce the power produced. However the maximum power produced by two and three connected cantilevers are still higher than maximum power for single $\mathrm{CL}$ of $272 \mu \mathrm{W}$ during $10 \mathrm{k} \Omega$ load resistance, because of its high boost in voltage output when connected in series. The matching load resistance for a single cantilever is $13 \mathrm{k} \Omega$. When it is connected in series connection with same polarity, matching load resistance for two connected cantilevers is $26 \mathrm{k} \Omega$, for three connected cantilevers it is $39 \mathrm{k} \Omega$, and for four connected cantilevers is $52 \mathrm{k} \Omega$. The cantilevers produce maximum power when connected to respective load resistance value.

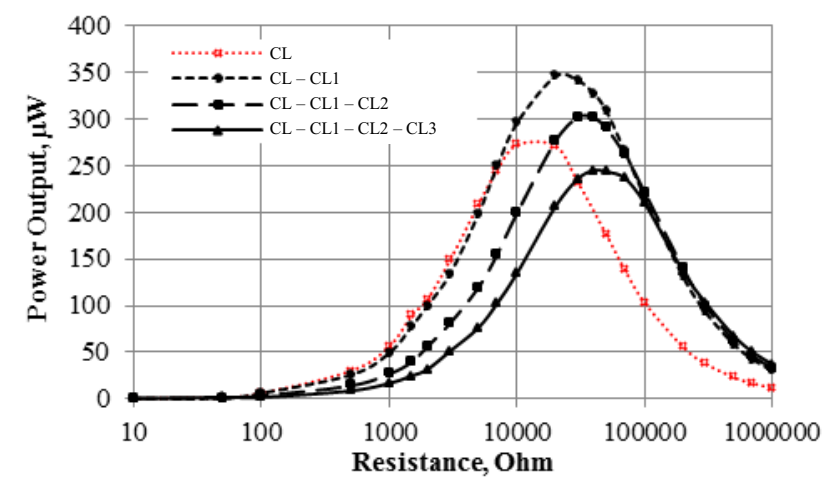

Figure 7. Output Responses (Power) toward Varies of Load Resistance for Cantilevers Connected in Series with Same Polarity Connection.

\subsection{Series Configuration with Alternating Polarities}

The series with alternating polarities connected cantilevers are observed under various resistances in this subsection. The result in terms of voltage output is shown in Fig. 8, while the result after converting to power value in shows in Fig. 9.

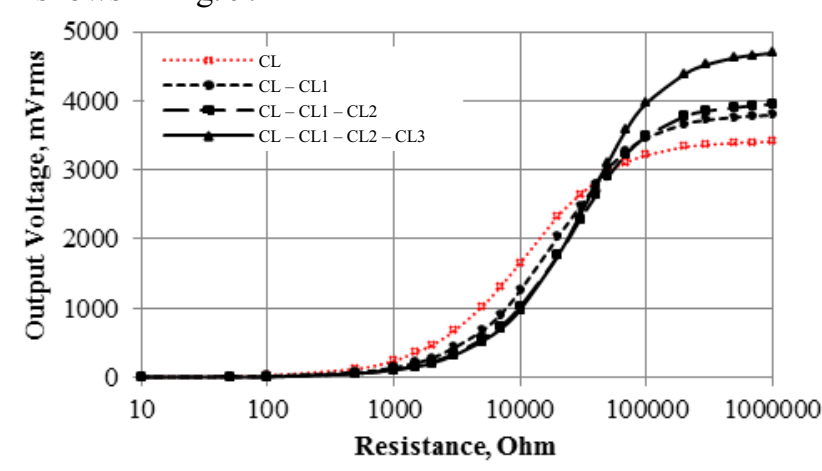

Figure 8. Output Responses (Voltage) toward Varies of Load Resistance for Cantilevers Connected in Series with Alternating Polarities Connection.

The result for voltage output is also similar to the result in Fig. 4, where the output increases slowly when low load resistance is connected to the cantilevers. It started to increase rapidly after $3 \mathrm{k} \Omega$ and starts to saturate when connected to a high load resistance. The maximum voltage outputs obtained from the multiple connected cantilevers are still higher than maximum voltage output produced from single $\mathrm{CL}$, which is $3.79 \mathrm{~V}$ for two connected cantilevers, $3.95 \mathrm{~V}$ for three connected cantilevers, and $4.69 \mathrm{~V}$ for four connected cantilevers. However, the voltage value is not as high as compared to the voltage result obtained from series with same polarities connection. This is due to the fact that 
piezoelectric cantilever generate output in alternating current (AC) form. Therefore, when connected in alternating polarities, the positive value of the output waveform is reduced by the negative value of the output waveform from the other cantilever.

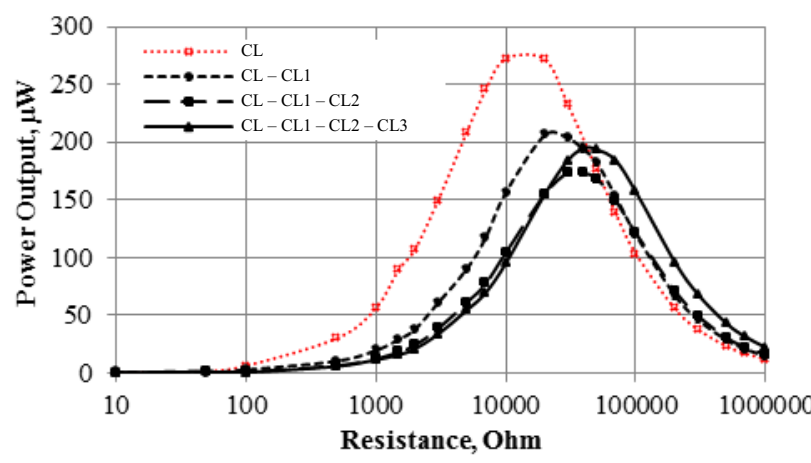

Figure 9. Output Responses (Power) toward Varies of Load Resistance for Cantilevers Connected in Series with Alternating Polarities Connection.

Same goes to its power output value, it is much lower compare to power produced by series with same polarities connection, in which two connected cantilevers produced maximum power of $206 \mu \mathrm{W}$ during $26 \mathrm{k} \Omega$ load resistance, three connected cantilevers produced maximum power of $173 \mu \mathrm{W}$ during $39 \mathrm{k} \Omega$ load resistance, and four connected cantilevers produced maximum power of $195 \mu \mathrm{W}$ during $52 \mathrm{k} \Omega$ load resistance. Notice that the matching resistances for piezoelectric cantilevers connected in series with alternating polarities connection are same as in series with same polarity connection.

\subsection{Parallel Configuration with Same Polarity}

As for parallel with same polarity connected cantilevers the result in terms of voltage output is shown in Fig. 10 while the result after converting it to power value in shown in Fig. 11.

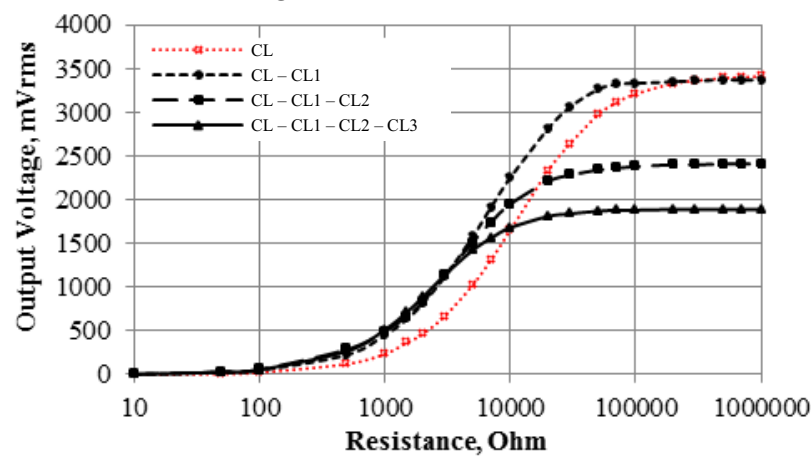

Figure 10. Output Responses (Voltage) toward Varies of Load Resistance for Cantilevers Connected in Parallel with Same Polarity Connection.

The voltage output also increased slowly at low load resistance and started to increase rapidly after $1 \mathrm{k} \Omega$ and starts to saturate when connected to high load resistance. The maximum output voltage achieved by two connected cantilevers is $3.37 \mathrm{~V}$, for three connected cantilevers is $2.41 \mathrm{~V}$, as for four connected cantilevers is $1.89 \mathrm{~V}$. When three other cantilevers are added to the connection, the total voltage output dropped because the voltage provided by cantilever CL1, CL2 and CL3 alone is lower than CL. Hence when average out, the total voltage output is decreased.

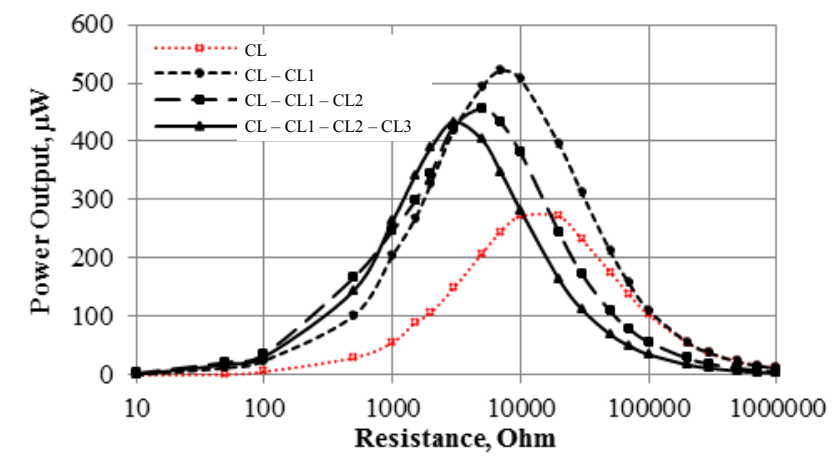

Figure 11. Output Responses (Power) toward Varies of Load Resistance for Cantilevers Connected in Parallel with Same Polarity Connection.

However, this is not the case for power output. The total power of the circuit was boosted up when power source are connected in parallel because current of the circuit increased when connected in parallel. This is shown in Fig. 11, where maximum power increased from $272 \mu \mathrm{W}$ to $521 \mu \mathrm{W}$ when two cantilevers are connected. However, the power drops to $456 \mu \mathrm{W}$ when three cantilevers are connected and to $433 \mu \mathrm{W}$ when four cantilevers are connected. This is due to the fact that total voltage output drops when connected in parallel. The increment in the current value is not high enough to cope with the dropping of voltage; hence the total power produced is lower. But still, the power outputs are higher than the output power produced by CL alone because of the current increased in parallel connection. The matching load resistances for two, three, and four connected cantilevers are $6.5 \mathrm{k} \Omega, 4.5 \mathrm{k} \Omega$ and $3.5 \mathrm{k} \Omega$ respectively since they reach maximum power when connected to those load resistance value.

\subsection{Parallel Configuration with Alternating Polarities}

The result in term of voltage output for parallel with alternating polarities connected cantilevers is shown in Fig. 12 while the result after converting to power value in shown in Fig. 13.

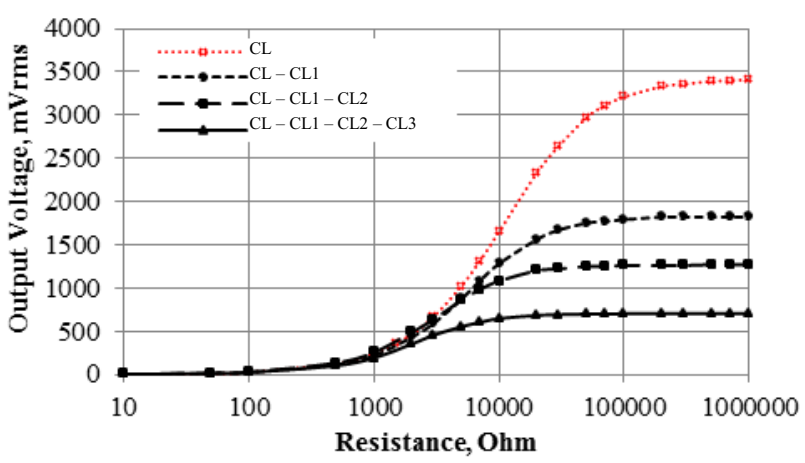

Figure 12. Output Responses (Voltage) toward Varies of Load Resistance for Cantilevers Connected in Parallel with Alternating Polarities Connection. 
Again the result shows output increased slowly when low load resistance is connected to the cantilevers and started to increase rapidly after $1 \mathrm{k} \Omega$ and starts to saturate when connected to high load resistance. Notice that the maximum voltage output produced in this case is much lower compared to the voltage output produced by CL alone. The maximum voltage output produced by two connected cantilevers is $1.82 \mathrm{~V}$, three connected cantilevers are $1.27 \mathrm{~V}$, and for four connected cantilevers is $0.71 \mathrm{~V}$. This is expected since voltage would drop for parallel branches. Furthermore, the cantilevers are connected in alternating polarities which means its positive value would be cancelled out by the negative value of the other connected cantilevers.

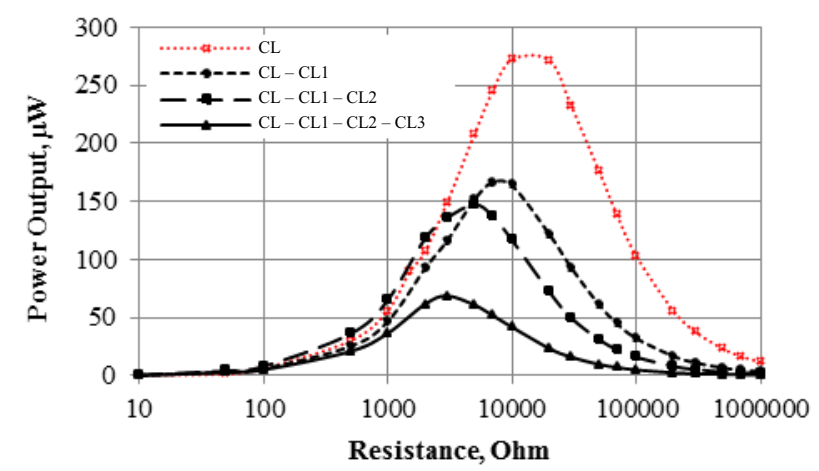

Figure 13. Output Responses (Power) toward Varies of Load Resistance for Cantilevers Connected in Parallel with Alternating Polarities Connection

Notice that the voltage value obtained in Fig. 12 for alternating polarities are much lower than the value obtained in Fig. 10 for same polarity. Even the converted power values shown in Fig. 13 are lower compared to power produced by cantilevers connected in parallel with same polarity connection. Where two connected cantilevers produced $167 \mu \mathrm{W}$, three connected cantilevers produced $147 \mu \mathrm{W}$, and four connected cantilever produced $69 \mu \mathrm{W}$. This is simply because the increased current value cannot cope with the decreased voltage value of the overall connected cantilevers.

In this case the matching load resistance for two connected cantilevers is still $6.5 \mathrm{k} \Omega$, for three connected cantilevers is $4.5 \mathrm{k} \Omega$, and for four connected cantilevers is $3.5 \mathrm{k} \Omega$.

\section{Conclusion}

The focus of this research is to investigate the changes in the output of the piezoelectric cantilever arrays when connected in different configuration. In term of voltage, all result shows that output voltage increase slowly during low load resistance, then increased rapidly after it reached certain resistance value, then starts to saturate at high load resistance. When converted to power values, the result shows that each array has one peak formed at respective matching load resistance, producing maximum power output. In series connections, the matching load resistance for two, three, and four connected cantilevers is $26 \mathrm{k} \Omega, 39 \mathrm{k} \Omega$ and $52 \mathrm{k} \Omega$ respectively. While in parallel connection, matching load resistance reduced to $6.5 \mathrm{k} \Omega$,
$4.5 \mathrm{k} \Omega$ and $3.5 \mathrm{k} \Omega$ for two, three, and four connected cantilevers respectively. These matching load resistance values are useful in order to maximize the output of the designed piezoelectric application. The resistance for the piezoelectric cantilever arrays posed the same behaviour as when $13 \mathrm{k} \Omega$ resistors are connected in series or parallel connection. The result also shows that when connected in series connection, the voltage output produced is much higher as compared to the piezoelectric cantilever arrays that are connected in parallel connection. The voltage output of the piezoelectric cantilever increased from $3.41 \mathrm{~V}$ to $6.09 \mathrm{~V}$ when it is connected in series configuration with same polarity. Whereas in term of power output, when connected to matching load resistance, piezoelectric cantilever arrays in parallel connection produce higher power output as compared to piezoelectric cantilever arrays in series connection. The maximum power increased from $272 \mu \mathrm{W}$ to $521 \mu \mathrm{W}$ when two cantilevers are connected in parallel configuration with same polarity.

\section{Acknowledgments}

The authors would like to thank the Ministry of Higher Education of Malaysia to sponsor this research project under the research grant no. FRGS/2/2014/SG02/FKEKK/02 as well as the facility support by the Advanced Sensors and Embedded Control System (ASECs) Research Group, Centre for Telecommunication Research and Innovation (CeTRI), UTeM.

\section{References}

1. Zhao, J., and You, Z., 2014. A Shoe-Embedded Piezoelectric Energy Harvester for Wearable Sensors. Sensor 2014, 14, pp.12497-12510.

2. Ansari, M.H., and Karami, M.A., 2015. Heartbeat Energy Harvesting Using the Fan-Folded Piezoelectric beam Geometry, In: Proceedings of ASME 2015International Design and Engineering Technical Conferences \& Computers and Information in Engineering Conference (IDETC/CIE 2015), Boston, Massachusetts, USA, 2-5 Aug 2015. ASME Publisher.

3. Aidin, D., and Jeremie, V., 2014. Flexible piezoelectric energy harvesting from jaw movements. Smart Materials and Structures, 23.

4. Yu, H., Zhou, J., Deng, L., and Wen, Z., 2014. A Vibration-Based MEMS Piezoelectric Energy Harvester and Power Conditioning Circuit. Sensors 2014, 14, pp.3323-3341.

5. Bong, Y.J., Kok, S.L. and Thong, L.W., 2014. Experimental Verification of Wide-Band Energy Harvesting using Piezoelectric Multi-cantilever with Resonant Frequency Variation. In: Malaysian Technical Universities Conference on Engineering and Technology (MUCET) 2014, Melaka, Malaysia, 10-11 November 2014. 
6. Gambier, P., Anton, S.R., Kong, N., Erturk, A., and Inman, D.J., 2012. Piezoelectric, solar and thermal energy harvesting for hybrid low-power generator systems with thin-film batteries. Measurement Science and Technology, 23.
7. Piezo Systems, Inc., 2011. Standard Quick-Mount Piezoelectric Bending Sensors (Generators). Available online: $<$ http://www.piezo.com/prodbg7qm.html $>$ [Accessed on 25 October 2016]. 